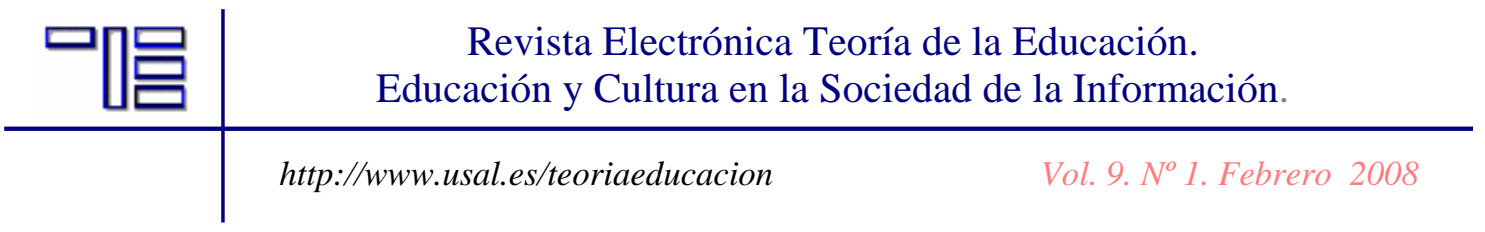

\title{
APRENDIZAJE ÉTICO EN CONTEXTOS VIRTUALES EN EL EEES.
}

Resumen: Se consideran algunos de los argumentos a favor de que la universidad integre entre sus prácticas de docencia y aprendizaje espacios que fomenten la formación ética y ciudadana de sus estudiantes. Se analizan como oportunidades para la mejora de la calidad y la renovación docente, el proceso de convergencia hacia el Espacio Europeo de Educación Superior y la progresiva integración de las tecnologías de la información, la comunicación y la documentación. Se identifican algunas de las características de las propuestas docentes que integran espacios virtuales de aprendizaje.

Se distinguen los siguientes ámbitos de aprendizaje y desarrollo de competencias transversales y de carácter ético en la universidad: contenidos curriculares, relaciones interpersonales, modelos de organización social del aprendizaje y evaluación de los aprendizajes de los estudiantes.

Se destacan conocimientos, habilidades, actitudes y valores que pueden ser aprendidos y movilizados por los estudiantes en los espacios virtuales de docencia-aprendizaje, en función de su contribución al desarrollo de competencias transversales y éticas en los estudiantes.

Palabras clave: Aprendizaje ético; valores; competencias transversales ; aprendizaje virtual; Espacio Europeo de Educación Superior.

\section{ETHICAL LEARNING IN VIRTUAL CONTEXTS IN THE EAHE.}

Abstract: The article considers some of the arguments in favour of the university incorporating systems that foster ethical and citizenship education in students into its teaching and learning practices. An analysis is made of the process of convergence towards the European Higher Education System and the progressive introduction of information, communication and documentation technologies as opportunities for improving quality and teaching renewal. Some of the characteristics of the teaching proposals that form part of virtual learning environments are also examined.

The article examines the following areas of learning and generic and ethical competencies in the university: curricular content, interpersonal relationships, models of social organisation of learning, and evaluation of students' learning processes.

It highlights the knowledge, skills, attitudes and values that can be learned and employed by students in virtual teaching-learning environments on the basis of their contribution to the development of students' generic and ethical competencies.

Key words: ethical learning, values, generic competencies, virtual learning, European Higher Education System

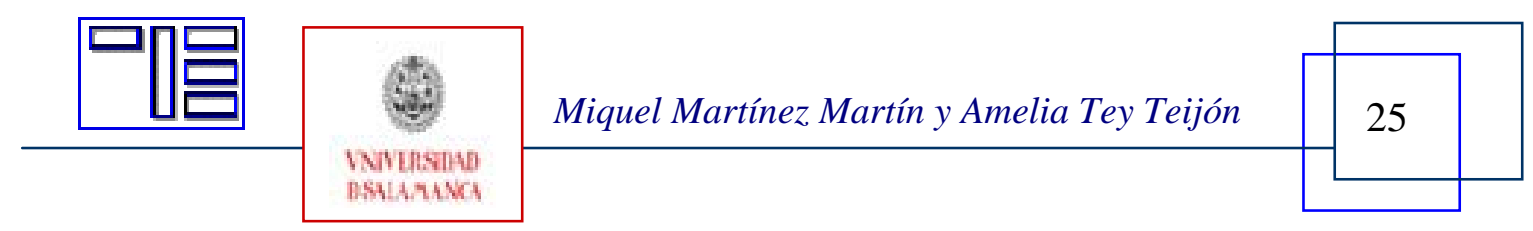


Revista Electrónica Teoría de la Educación.

Educación y Cultura en la Sociedad de la Información.

http://www.usal.es/teoriaeducacion

Vol. 9. No 1. Febrero 2008

\section{ÉTHIQUES APPRENTISSAGE DANS LES CONTEXTES VIRTUELS DANS L'EEES.}

Sommaire: On considère certains arguments qui défendent que l'Université intègre, dans les pratiques d'enseignement apprentissage, des espaces qui favorisent la formation éthique et citoyenne de ses étudiants. On analyse comme une opportunité pour l'amélioration de la qualité et le renouveau de l'enseignement le processus de convergence vers l'espace européen de l'éducation supérieur et la progressive intégration des nouvelles technologies de l'information, la communication et la documentation. On identifie certaines caractéristiques des propositions d'enseignement qui intègrent des espaces virtuels d'apprentissage.

À l'université, on distingue les suivant domaines dans le cadre de l'apprentissage et le développement des compétences transversales et de caractère étique: les contenus du curriculum, les relations interpersonnelles, les modèles d'organisation sociale de l'apprentissage et l'évaluation des apprentissages des étudiants.

On souligne les connaissances, les habilités, les attitudes, les valeurs qui peuvent être apprises et mobilisées par les étudiants au travers d'espaces virtuels de l'enseignement apprentissage, en fonction de sa contribution au développement des compétences transversales et étiques chez les étudiants.

Mots clés : apprentissage étique, valeurs, compétences transversales, espace européen de l'éducation supérieur, apprentissage virtuel

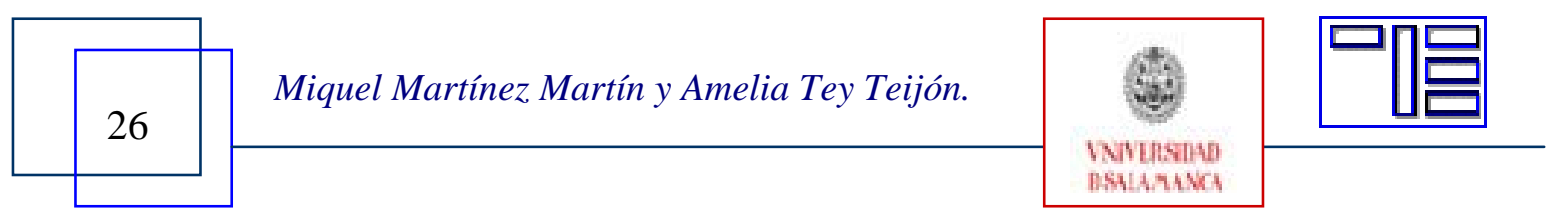




\title{
APRENDIZAJE ÉTICO EN CONTEXTOS VIRTUALES EN EL EEES.
}

\author{
Miquel Martínez Martín y Amelia Tey Teijón
}

miquelmartinez@ub.edu, atey@ub.edu

Universitat de Barcelona

\section{LAS UNIVERSIDADES Y LA FORMACIÓN ÉTICA DE SUS ESTUDIANTES.}

La progresiva convergencia que genera la construcción del Espacio Europeo de Educación Superior ayuda a plantear desde nuevas perspectivas algunos de los clásicos compromisos que las universidades formulan con frecuencia a modo de declaraciones de intenciones en sus documentos constituyentes, estatutos o planes estratégicos. Uno de ellos consiste en la formación integral de sus titulados; es decir, no sólo como profesionales, investigadores o profesores, sino también como ciudadanos. Ahora bien, este compromiso que habitualmente es así formulado, es olvidado con frecuencia en las propuestas docentes que conforman el modelo formativo de las diferentes titulaciones.

En otras ocasiones ya hemos argumentado la importancia de la formación ética y ciudadana de los universitarios, como factor de calidad de la educación superior en el mundo actual y en concreto en el marco de las declaraciones que a partir de la de Berlín están configurando el proceso de construcción del espacio europeo. En dichos escritos hemos identificado tres dimensiones (Martínez, 2006) en la función ética de la universidad en la sociedad actual. Una, la formación deontológica relativa al ejercicio de las diferentes profesiones. Dos, la formación ciudadana y cívica de sus estudiantes. Y tres, la formación humana, personal y social, que contribuya a la optimización ética y moral de las futuras y futuros titulados en tanto que personas. En relación con estas tres dimensiones puede afirmarse que la primera está razonablemente aceptada como un signo o indicador de calidad, la segunda es aceptada de forma progresiva como una necesidad en cuyo logro la universidad debe colaborar y, la tercera, como algo que a excepción de algunas universidades, corresponde a otros niveles del sistema educativo y que difícilmente puede integrarse en la misión y función de las universidades.

En general, y al margen de declaraciones y documentos, el profesorado universitario es ajeno a estas preocupaciones y tiene cierto recelo a la hora de plantear la formación ciudadana y en valores democráticos como algo inherente a la formación universitaria. Entre otras razones porque entiende tal y como hemos manifestado anteriormente que, incluso reconociendo la importancia de tal formación, la responsabilidad no es suya. Cada vez más reconoce la importancia de la formación deontológica de sus estudiantes para que en el futuro puedan desarrollar un buen ejercicio profesional, pero rara vez se plantea entre sus objetivos docentes la formación ética dirigida a la construcción de la personalidad moral y a la formación ciudadana de sus estudiantes. Consecuentemente,

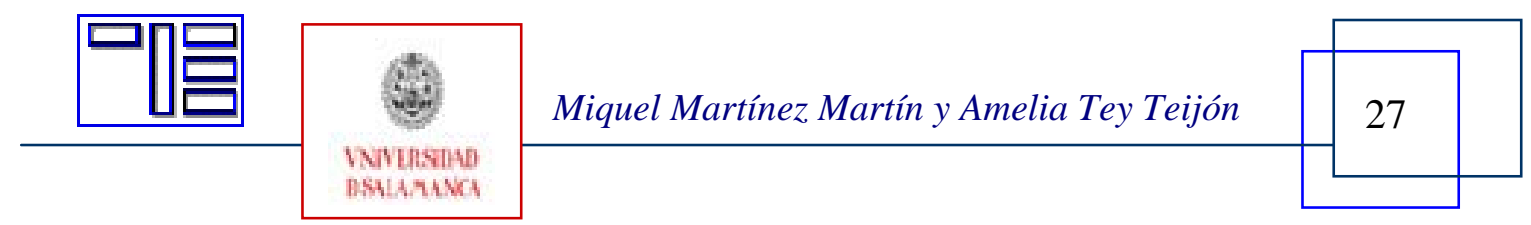


tampoco dedica ni el tiempo ni el espacio necesario para identificar qué competencias y qué resultados de aprendizaje en concreto deben integrarse en los planes docentes y en las prácticas de aprendizaje en la universidad.

El término aprendizaje es fundamental en nuestro planteamiento, puesto que al margen de la voluntad explicita y consciente del profesorado, los contextos de convivencia, estudio, ocio y aprendizaje que los estudiantes viven en su periodo universitario son potencialmente espacios de aprendizaje ético. Esta es la razón por la que nos referimos a la universidad como un buen "lugar" donde aprender éticamente.

El mundo de los valores se "aprende" en la cotidianeidad del aprender y el convivir, y la universidad es, valga la redundancia, un lugar de aprendizaje y convivencia. Pero incluso en el caso en que coincidiéramos en que la universidad es un espacio adecuado, conviene que reúna algunas condiciones para que sea un espacio eficaz. Podemos proponernos que la universidad sea un espacio de construcción de valores en el que los estudiantes que en ellas se forman puedan construir sus propias de matrices de valores guiados por ideales como la dignidad, la libertad y la justicia de forma que mediante el ejercicio de sus profesiones contribuyan a transformar nuestra sociedad avanzando en una mayor inclusión social y profundizando en los valores de la democracia participativa. Pero no lo conseguiremos si limitamos la función ética de la formación universitaria a una función de transmisión, instrucción y reproducción de tales valores.

Para que el aprendizaje en la universidad suponga también aprendizaje ético y éste sea eficaz es necesario construir espacios en los que tales valores estén presentes de forma habitual, cotidiana y natural. No todas las formas de enseñar y aprender son adecuadas para apreciar determinados valores y construir matrices de valores personales orientadas a la consolidación de estilos de vida personales y comunitarios, activos y democráticos. Las condiciones de aprendizaje y docencia y entre ellas las que afectan a la tipología de contextos presenciales y virtuales en la universidad actual pueden contribuir al desarrollo y consolidación de competencias transversales catalizadoras de prácticas de aprendizaje que contengan valores.

\section{EL ESPACIO EUROPEO DE EDUCACIÓN SUPERIOR Y LAS PROPUES- TAS DOCENTES QUE INTEGRAN ESPACIOS VIRTUALES.}

La construcción del EEES propicia espacios de reflexión y de elaboración compartida de propuestas docentes que atienden a diferentes cuestiones. Entre éstas: una concepción del aprendizaje centrada en el estudiante que reubica la tarea del profesorado; la fijación de objetivos docentes en términos de competencias y la determinación de los resultados de aprendizaje que esperamos alcancen los estudiantes; el establecimiento de modelos de evaluación continua y el aprovechamiento óptimo de las tecnologías para el aprendizaje. En relación con todas estas cuestiones podemos encontrar elementos que permiten una reflexión desde el mundo de los valores sobre como las formas de aprender, enseñar y evaluar en la universidad influyen éticamente en los estudiantes y en su

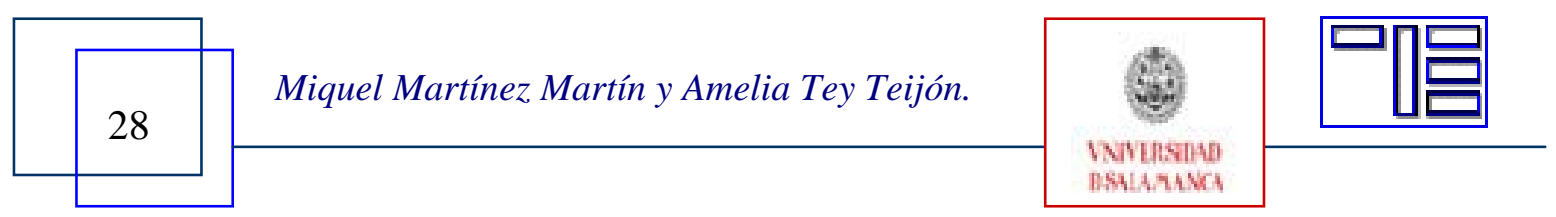


formación. En lo que sigue nos referiremos a las que están relacionadas con la naturaleza virtual de los contextos de aprendizaje.

La consideración de las tecnologías de la información, comunicación y documentación en los procesos de aprendizaje y docencia en la universidad es una realidad ampliamente extendida. Obviamente no todas las universidades han alcanzado al respecto el mismo nivel de desarrollo ni lo han integrado siempre de manera eficaz ni de la misma forma en sus procesos de aprendizaje y docencia. Sin embargo es un hecho que la mayoría de universidades que están revisando sus propuestas docentes lo hacen integrando docencia, consulta, debates y foros presenciales con foros y docencia no presencial. Esta combinación o mezcla -blend- supera la dicotomía presencial-virtual que en los últimos años ha diferenciado las universidades clásicas de las universidades a distancia o virtuales. Hoy en día es difícil sostener un modelo exclusivamente presencial o exclusivamente virtual. Prácticamente sólo la tipología de los estudiantes, alejados del centro universitario o con poca disponibilidad horaria para poder asistir regularmente de forma presencial, es la que motiva sistemas virtuales en sentido estricto. Y sólo una limitación en recursos tecnológicos y en formación del profesorado explica porqué en algunas universidades no se ha iniciado aún la armonización entre lo presencial y lo virtual.

Por otra parte, la sociedad actual ha creado nuevas formas de alfabetización -incluimos aquí la digital- a las que no es ajena la formación universitaria, mediante la que también es posible el desarrollo de competencias ciudadanas (Gros; Contreras 2006).

Cada práctica alfabetizada tiene un formato determinado. No es lo mismo leer un artículo, una revista, un libro, o un documento de internet, por citar algunos de los ejemplos más habituales. En cada una de estas prácticas la persona debe estar familiarizada con el desempeño de ciertas rutinas y actividades específicas, que denotan el dominio de las competencias necesarias para el caso. El dominio de cada práctica exige unos conocimientos, habilidades y actitudes concretas y diferentes entre sí.

\section{LOS ESPACIOS VIRTUALES DE APRENDIZAJE.}

No obstante, la convergencia entre todas estas prácticas se manifiesta en la finalidad de su uso, puesto que en todas ellas, sea cual sea su formato y estructura, el lector se apropia del texto o contenido de una manera personal. Al referirnos a la alfabetización no podemos hacerlo, pues, pensando en algo cerrado, inamovible e universal sino, por el contrario, concibiéndola como una práctica dentro de un proceso, como una actividad del sujeto que es significativa, le transforma, y que, además, transforma la realidad (Rodríguez,2005,15).

Cualquier novedad genera interés a la vez que cierta inquietud por el uso, mal uso o abuso, que se haga de ella. Es un recelo que en ocasiones hemos detectado también cuando en la universidad se plantean los entornos virtuales como espacios para el aprendizaje. No obstante, confirmamos por propia experiencia que es un recelo se dilu-

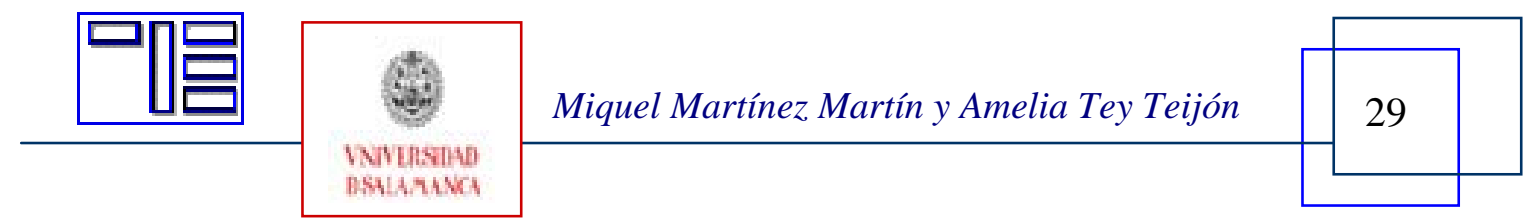


ye cuando, tras el conocimiento y utilización del mismo, se perciben como espacios abiertos de no presencialidad pero en los que se favorece el aprendizaje interactivo, y espacios asincrónicos que favorecen la flexibilidad en el proceso de aprendizaje.

El encuentro entre extraños es un acontecimiento sin pasado (Bauman, 2000), pero contrariamente a la visión del autor, que afirma que frecuentemente también es un acontecimiento sin futuro, pensamos que el encuentro en espacios virtuales de aprendizaje también puede tener futuro. Tales espacios pueden generar las condiciones no sólo para establecer una comunicación instrumental con el tutor y los compañeros, si no para convertirla en una relación auténtica (Taylor, 1994) y sincera.

La formación basada en la red la definimos, de acuerdo con Luis Cabero, como una modalidad formativa a distancia que se apoya en la red y que facilita la comunicación entre el profesor y los alumnos según determinadas herramientas sincrónicas y asincrónicas de la comunicación (Cabero, 2006,2). La sincronía se manifiesta al usar determinadas herramientas en las que los participantes no están físicamente juntos pero sí temporalmente -éste seria el ejemplo del chat-, mientras que la asincronía implica una destemporalización de la actividad formativa de los participantes. Es así porque uno de los rasgos que más rápidamente identificamos de la formación virtual es que permite la deslocalización del conocimiento y, consecuentemente, que los estudiantes vayan a su propio ritmo de aprendizaje. Ejemplos de ello serían el trabajo en aula Moodle, el uso del knowledge forum o las wikies. La virtualidad rompe y supera las barreras espaciotemporales y la posibilidad de "conectar" con los contenidos en el momento más adecuado para el participante facilita en gran medida que éste compagine su formación con otras dedicaciones -profesionales y/o personales-. Podemos, pues, afirmar que el aprendizaje en contextos virtuales se basa en el concepto de la formación en el momento en el que se necesita “just-in-time tranining” (Cabero, 2006).

Los espacios virtuales de aprendizaje configuran un nuevo escenario en el que las reglas, las maneras de hacer, de relacionarse, de comunicarse y de trabajar son diferentes. La relación que establece el profesor y el estudiante facilita que el conocimiento sea un proceso activo de construcción, y aunque el proceso de aprendizaje tiende a realizarse de manera individual, ello no quiere decir -como comentaremos posteriormente- que se renuncie a propuestas de aprendizaje colaborativas (Cabero, 2006). Estudiantes y profesorado establecen nuevos roles, los primeros mucho más autónomos en su proceso de aprendizaje y los segundos a través de una praxis mucho más cercana a la de creador de condiciones educativas y diseñador de experiencias pedagógicamente potentes para favorecer el aprendizaje.

Los espacios virtuales de aprendizaje, de la misma manera que los espacios de aprendizaje presencial, son resultado de la interacción entre personas, de origen muy diverso, unidas por el interés por la formación. Son personas capaces de sentir y de manifestarse, es decir, son sujetos éticos. Se presentan en el contexto virtual con una matriz de valores

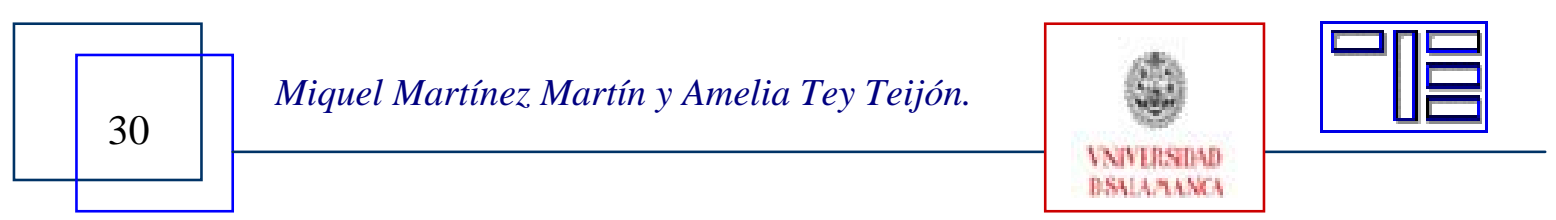


determinada y, de la misma manera que en otros contextos, siguen considerando como moralmente relevantes ciertas situaciones, como correctas determinadas reacciones y no están de acuerdo con otras. Pero, paradójicamente, si los valores son los mismos, puede modificarse la manera de expresar su moralidad. Conforman un tejido social en el que se comparten vivencias, experiencias y conflictos, y también se expresan emociones y sentimientos que no sustituyen la relación presencial sino que la complementan (Duart,2003). En definitiva, los espacios virtuales de aprendizaje son creaciones artificiales en las que pueden generarse prácticas ricas en valores o representativas de contravalores.

El tercer entorno (Echevarría, 2000), también en la universidad, no es sólo un nuevo espacio de información y comunicación sino también un espacio social para la interacción, la memorización, el entretenimiento y la expresión de emociones, entre otras cosas. Por todo ello, el complejo tejido social que se conforma y el entramado de relaciones que se configura realzan la importancia de la presencia ética en los espacios de formación virtual. Los entornos sociales favorecen que la persona muestre sus valores a través del comportamiento; pero a la vez, la naturaleza dinámica del desarrollo explica por qué la reestructuración de los espacios puede influir en la configuración moral de la persona. (Tey, 2004,84; 2005).

Nuestro objetivo es favorecer que el estudiante universitario sea y se sienta competente éticamente y, por lo tanto, lograr que su participación en contextos comunitarios y en la toma de decisiones sea ponderada, pertinente y justa. Es precisamente la consideración de estas competencias la que influirá en la mirada del profesorado al valorar los ámbitos que en la formación universitaria son susceptibles de generar aprendizaje ético.

En otros momentos hemos distinguido al respecto dos tipos de competencias, las de carácter específico -relacionadas con las competencias para el ejercicio de la ciudadanía y con posibilidad de desarrollarlas desde una materia concreta - y las de carácter genérico -o competencias éticas- (Buxarrais, Tey 2006). Las competencias éticas pueden desarrollarse desde muchas materias y deben permitir la construcción personal de un modelo de vida buena y justa y actuar con criterio en cuestiones o situaciones moralmente controvertidas. Son competencias fundamentales para comprender críticamente nuestro mundo, gestionar cambios que contribuyan a su transformación y mejora, actuar responsablemente, comprometerse e implicarse en proyectos; y en definitiva, sentirse actor en su vida y agente activo en la comunidad. Son competencias transversales que integran competencias instrumentales, interpersonales y sistémicas de acuerdo con la relación de competencias del proyecto Tuning.

Las competencias éticas, como toda competencia, implican capacidad para movilizar de forma adecuada recursos cognitivos- conocimientos, habilidades y actitudes- y para abordar de forma eficiente situaciones concretas. Son competencias que añaden al saber y a los conocimientos en particular, el saber movilizarlos en un momento concreto y de forma adecuada. De ahí que los entornos virtuales de aprendizaje sean buenos espacios

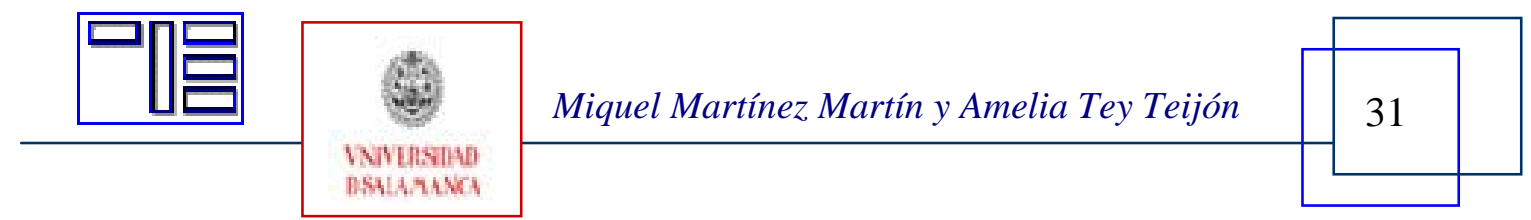


para su desarrollo y manifestación. Son competencias que pueden aprenderse y mostrarse tanto en situaciones de interacción como en la ejemplificación de aprendizajes relativos a las diferentes materias de una titulación. Por su relevancia y amplitud, y por ser precisamente genéricas y transversales, es importante desarrollarlas en el conjunto de toda la titulación y no en una materia en concreto. Pero para ello el profesorado debe ser consciente de que es posible, y también valorar la importancia que tiene una buena planificación y evaluación de las competencias transversales en las propuestas docentes de las titulaciones.

\section{4. ÁMBITOS DE APRENDIZAJE Y DESARROLLO DE COMPETENCIAS TRANSVERSALES Y DE CARÁCTER ÉTICO.}

Las competencias éticas deben ser objetivos de la formación universitaria, puesto que contribuyen al desarrollo del estudiante en tanto que persona, profesional y ciudadano (Martínez, Buxarrais, Esteban,2002); y pueden desarrollarse a partir de su consideración en los contenidos curriculares, las relaciones interpersonales, los modelos de organización social del aprendizaje y en la evaluación.

\section{A) Contenidos curriculares}

Compartimos con Julio Cabero que los contenidos curriculares deben analizarse desde una triple perspectiva: cantidad, calidad y presencia. Por una parte, es fundamental establecer la cantidad justa de contenidos en función de los objetivos de formación y las características del grupo y del formador. Pero además consideramos necesario matizar que la selección de los contenidos curriculares no está exento de valor ético, y que ello supone un cambio de mirada del profesorado cuando planifica su asignatura, se plantea los objetivos, las fuentes de información y la selección de las actividades (Martínez, 2006). La presentación y el abordaje de situaciones moralmente relevantes y complejas éticamente, favorecen que el estudiante sea capaz de analizar mejor temas y situaciones afines a las que tendrá que dar respuesta como profesional y como persona. Permiten que perciba la controversia como una realidad y progresivamente sea capaz de no expresar un pensamiento únicamente lineal sino analizar las variables de aquello que es “complejo" para establecer relaciones convergentes, paralelas y/o divergentes. Los contenidos son la excusa para generar una mayor sensibilidad moral y, en la misma medida, para el desarrollo de la reflexión sociomoral, el razonamiento y el análisis crítico desde la complejidad de la situación.

Para ello el profesorado tiene que asegurar un elevado nivel de calidad en los contenidos que presenta, conjugando la pertinencia, la relevancia y la actualización de los mismos. Así es posible estimar el rigor en el tratamiento de la información y en general en la construcción del conocimiento científico, y potenciar actitudes concretas en los estudiantes y como futuros profesionales relativas al interés por saber más, contrastar diver-

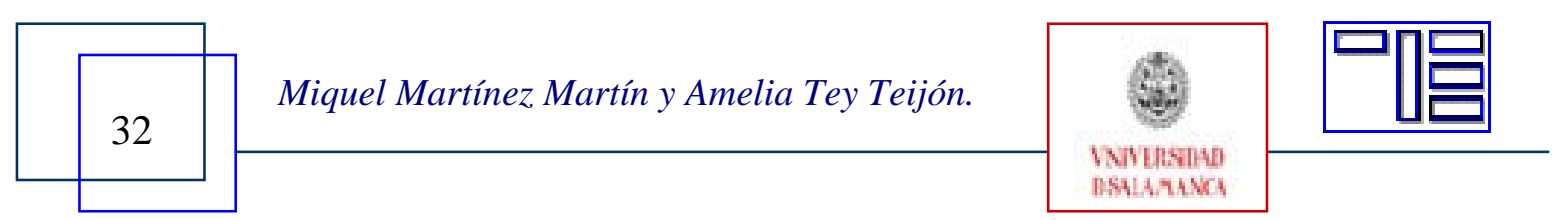


sas fuentes de información , discriminar las menos profundas y seleccionar las más pertinentes.

Por último, pero no por ello menos importante, conviene considerar el diseño, la estructuración y presentación de los contenidos en la plataforma, elemento clave para favorecer la accesibilidad de los participantes y motivar su uso constante (Cabero, 2006). Este puede ser un modelo a imitar, por su calidad en la forma de planificar y disponer la información para el aprendizaje, que puede potenciar actitudes y valores en la forma de aprender del estudiante y en la de ejercer en el futuro su profesión.

\section{B) Relaciones interpersonales}

El comportamiento digital convierte a todas las personas participantes en modelos para los demás. Lo mismo sucede con el profesorado, al que además se le atribuye comunitariamente el rol educativo. De él se espera un comportamiento moral -atento y justo- en su relación con los estudiantes (Katz, Noddings, Strike, 2002) puesto que, de la misma manera que lo es en la formación presencial, también es modelo para los estudiantes cuando se comunica con ellos a través de la plataforma virtual. Al referirnos a la comunicación en la relación debemos diferenciar la relación docente y la interpersonal, que se establece paralelamente de la anterior. En la primera el rol del profesor como experto, asesor y tutor académico impide la situación de simetría que, por el contrario, sí que observamos en el segundo tipo de relación (Martínez, 2006).

El problema con la tecnología digital es que las reglas para su uso no se han escrito todavía y, los usuarios no han tenido la oportunidad de ponerse al día con la forma adecuada de usarlas. Algunas de las reglas o políticas se presumen, mientras que otras han sido creadas por usuarios individuales o por grupos de éstos.

El profesor debe tenerlo claro pero lo realmente complicado es que son los estudiantes los que deben aprender a diferenciar cuál es la forma de comunicación más adecuada para una circunstancia determinada (Tey, 2004). Es así, porque aunque los procesos de socialización también se realizan virtualmente, éste es un proceso más complicado al comprobar que, citando a Yus y extraído de Prats, pocas veces comunicamos de manera literal, sino que "dejamos implícita toda aquella información que prevemos que nuestro interlocutor podrá obtener por sí mismo, por lo que la tarea de acceder a la interpretación pretendida por el emisor puede llegar a ser un proceso inferencial bastante complicado" (Yus, 2001; Prats, 2005,5). La necesidad de llegar a un elevado nivel de competencia comunicativa y argumentativa debe pues complementarse con una visión anticipatoria de lo que pueden ser las reacciones del interlocutor. Las habilidades sociales y el diálogo, como herramienta y también como valor, devienen habilidades facilitadoras de relación y aprendizaje.

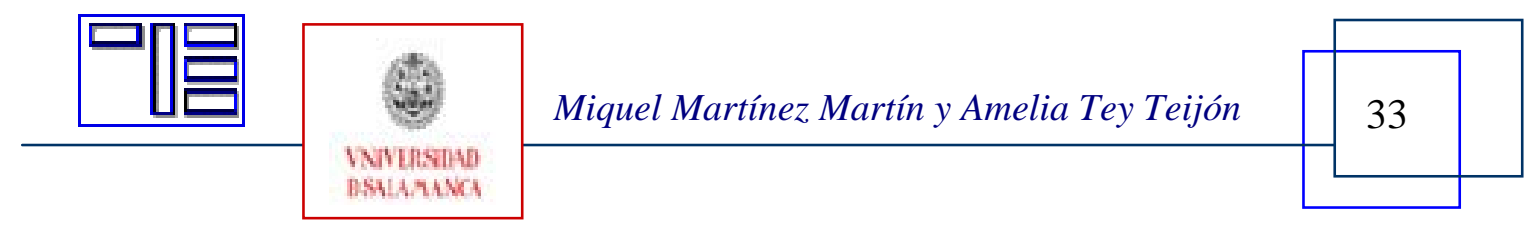


Al diseñar una actividad de aprendizaje virtual deberíamos considerar, como mínimo, tres elementos: las capacidades cognitivas y lingüísticas, la consideración del contexto, y su valoración crítica. El conocimiento y comprensión del funcionamiento técnico es esencial, puesto que es la herramienta que utilizamos; pero el lenguaje, es la vía de comunicación y también el soporte interno con el que se sustenta lo técnico, por lo que para poder contribuir a la formación de los estudiantes debemos asegurar un lenguaje compartido en la intersubjetividad. De ahí que debamos añadir a las competencias cognitivas y lingüísticas, que apuntábamos anteriormente, el conocimiento de las características del contexto, el uso que se le da y las consecuencias sociales y personales de dicho uso. Citamos literalmente a Prats cuando afirma que "el ciberespacio es una recreación humana (un artefacto, en suma) que quiere imitar lo natural para trasgredirlo y superarlo” (Prats, 2005). Precisamente esta idea es la que sustenta las muestras de capacidad crítica para pensar las practicas digitales como practicas socialmente construidas. (Rodríguez, 2005, 18)

Una de las características que se desprenden del aprendizaje virtual es que la naturaleza de la formación modifica las formas de organización social sobre las tareas de aprendizaje. La existencia del aula virtual imprime un sentido propio a las actividades que allí se llevan a cabo y desplaza el centro de interés de la enseñanza al aprendizaje, y, de la misma manera, debería trasladar el centro del eje de la información unilateral a la interacción. Aprender y hacer son acciones inseparables, nos dice Begoña Gros, pero para ello los estudiantes deben aprender en el contexto pertinente. De ahí que el diseño de los contextos de aprendizaje se convierte en uno de los pilares de la formación virtual (Gros, Contreras, 2006).

La formación virtual favorece la construcción del conocimiento al convertir al participante en un agente totalmente activo en su proceso de aprendizaje, en el que "las representaciones individuales y las actividades sociales culturalmente organizadas están íntimamente interconectadas y se influyen mutuamente manteniendo, en palabras de Salomón $(2003,192)$ una relación fluida y "transaccional”, por lo que ambas deben ser tenidas en cuenta para comprender los procesos de construcción del conocimiento que llevan a cabo los estudiantes” en las aulas convencionales (Coll,1990, 162 ) y también en las aulas virtuales.

No podemos dejar de mencionar las comunidades virtuales, como una de las formas de organización social típicas de la formación virtual. Uno de sus rasgos definitorios es la

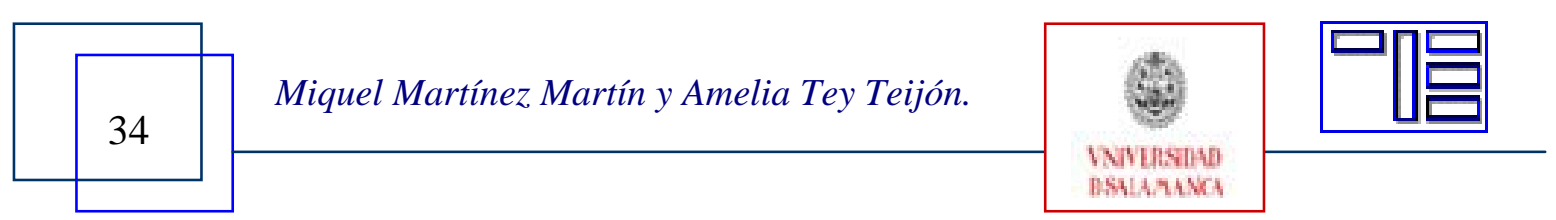


búsqueda de la construcción de conocimiento social (Garcia, 2007), para lo que se favorece la división de tareas y el conocimiento personal, que posteriormente se socializa en el software para el trabajo en grupo, por lo que se enriquece al ponerlo en común.

Crook propone quatro formas de interacción relacionadas con los ordenadores, de entre las que destacamos interacciones colaborativas alrededor de los ordenadores y a través de ellos, porque en este caso los participantes pueden colaborar separados en el tiempo y en el espacio (Crook, 1998). De esta manera en la comunidad virtual se entremezcla la autoformación con el interés por compartir información, veraz y completa. La ética de la información se pone en juego en la comunidad virtual. En otro escrito ya apuntábamos a la objetividad, la autenticidad, la veracidad, la oportunidad y la pluralidad como las principales líneas éticas en la gestión de la información ya que cuando no se consideran se falta a uno de los derechos fundamentales de los ciudadanos en la transmisión de la información (Tey, 2004). La ética de la información refleja pues derechos, pero también obligaciones de todos los usuarios de la formación virtual. Nos referimos tanto a las intervenciones y productos elaborados por los participantes como en el desempeño del profesor, que pasa ser un facilitador del proceso.

Las formas de organizar las actividades, bien estructuradas, pueden generar un tipo de interacciones, de relaciones y un sentimiento de reciprocidad entre profesor y alumno, y también entre estudiantes, en el que cada uno sabe lo que se espera del otro. Se espera fundamentalmente respeto y responsabilidad, puesto que son los valores que permiten mantener las reglas del juego tal y como las estamos presentando. De ahí deriva que, en el modelo que proponemos, apostemos por actividades que rehuyan de un proceso de elaboración meramente individualista y/o memorístico. En cambio potenciaremos aquellas actividades en las que los participantes dialoguen, reflexionen conjuntamente, colaboren y elaboren objetivos para la resolución de las actividades. Son actividades en las que el trabajo en equipo es fundamental pero en las que, paralelamente, exigiremos la manifestación de la toma de posición fruto de lecturas y de reflexión personal. A la vez, son actividades que favorecen que el estudiante consiga progresivamente niveles superiores de autorregulación, tanto en la gestión del tiempo, como en la actitud serena y responsable hacia el esfuerzo que implica la transformación de ciertas informaciones en conocimientos $y$, consecuentemente, el aprendizaje.

\section{D) Evaluación continua}

El aprendizaje autónomo y la implicación del estudiante en su propio proceso imprime a la formación virtual un sello particular y diferenciador de los procesos de formación estrictamente presenciales. La evaluación se contempla, en este caso, como un elemento más, configurador de todo el proceso al que estamos aludiendo. Concebida así, no es de

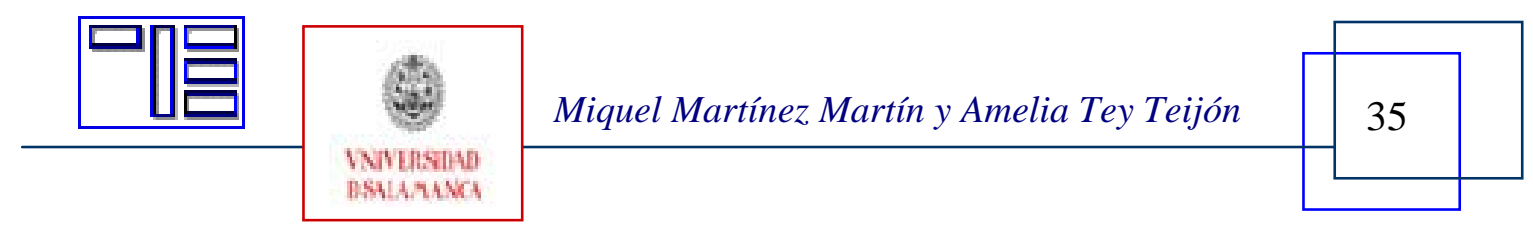


extrañar que se plantee como una evaluación continua a partir de la cual podamos asegurar su función acreditativa, formativa y formadora ${ }^{1}$.

Se parte de la base que es el estudiante quien construye su aprendizaje, por lo que debe implicarse y esforzarse para conseguir su optimo resultado. La responsabilidad del propio estudiante en relación con sus acciones permite una mayor o menor aprendizaje en función del proceso de autorregulación. El profesor facilita los recursos, genera las condiciones y acompaña en el proceso pero es el propio estudiante quien decide cómo y cuándo y cuánto leer y estudiar.

La evaluación continua posibilita gestionar y modificar la dedicación y el esfuerzo de los participantes y proporciona informaciones y criterios para autorregularse, a través de diversos feed-backs al estudiante, que en última instancia se responsabiliza de sus acciones.

Iniciábamos el artículo oponiéndonos a las posiciones que perfilan la formación en entornos virtuales de aprendizaje como generadoras de actitudes individualistas y competitivas. Hemos analizado los contenidos, las relaciones interpersonales, la organización social, y los modelos de evaluación, concebidos como espacios capaces de generar condiciones que favorezcan aprendizaje ético en los estudiantes. Nos hemos referido a conocimientos, habilidades, actitudes y valores que los espacios virtuales de aprendizaje pueden generar y movilizar. Es así que los espacios virtuales pueden generar el desarrollo de competencias transversales de carácter ético en los estudiantes.

Algunas de estas competencias están relacionadas con las que forman parte de la relación de competencias transversales - instrumentales, interpersonales y sistémicas- del proyecto Tunning. A modo de ejemplo y entre las competencias instrumentales: la habilidad de gestión de la información, la capacidad de organización y planificación, comunicación oral y escrita en la propia lengua y habilidades básicas del manejo del ordenador entre otras. Entre las competencias interpersonales: la capacidad de crítica y autocrítica, el trabajo en equipo, la apreciación de la diversidad y multiculturalidad, el compromiso ético y la habilidad para trabajar en un contexto internacional. Por último, y entre las ccompetencias sistémicas: trabajar de forma autónoma, con capacidad para adaptarse a nuevas situaciones de manera creativa y desarrollo de motivación de logro; el diseño y gestión de proyectos en los que puede trabajar con personas de otros países, la preocupación por la calidad de sus procesos y productos.

Por último y como conclusión queremos destacar los dos tipos de aprendizaje que los espacios virtuales fomentan mediante la práctica y que desde nuestro punto de vista son factores de aprendizaje ético. Son practicas de aprendizaje que desarrollan competencias que contribuyen a que la persona se más autónoma y a la vez más cooperativa y colaborativa. En definitiva la hacen moralmente más competente.

\footnotetext{
${ }^{1}$ En este apartado seguimos las propuestas elaboradas desde el Vicerrectorado de Política Docente de la Universitat de Barcelona para la aplicación de las Normas Reguladoras de la evaluación aprobadas por el Consejo de Gobierno del 6 julio de 2006.
}

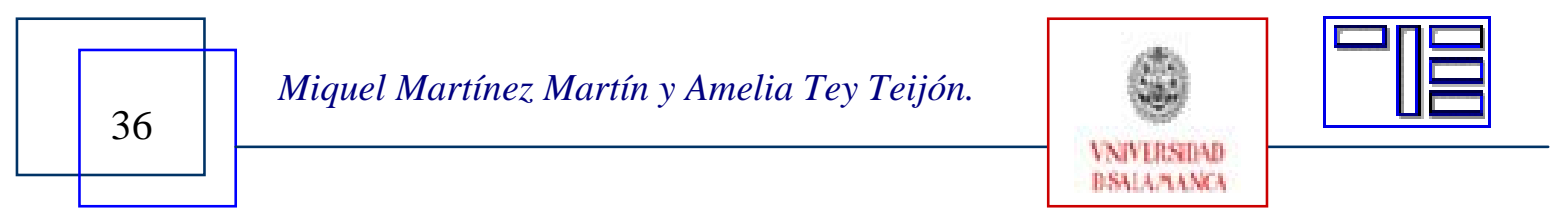




\section{Aprendizaje autónomo}

Una de las premisas fundamentales para que la formación a distancia revierta realmente en un aprendizaje del estudiante es que muestren competencias personales para el aprendizaje autónomo. En las actividades de formación virtual el estudiante asume un rol mucho más activo en su propio proceso de aprendizaje y se convierte en agente promotor de la transformación de la información en conocimiento, a partir de un proceso de aprendizaje mucho más significativo para él. A la vez, el aprendizaje autónomo favorece que la persona se sienta competente para aprender a lo largo de la vida. (Sigalés, 2004)

Desaparece la memorización de la información y se centra en la construcción del conocimiento a partir de la reestructuración de la información (Coll, 1990).

La formación virtual pone a disposición del estudiante un amplio volumen de información, pero son ellos quienes la convierten en conocimiento. Puede llegar a ser un inconveniente que, requiere más inversión y tiempo por parte del profesor y los estudiantes, como mínimo en los primeros estadios de la formación virtual, para llegar a desarrollar las habilidades para el aprendizaje autónomo.

Aprender autónomamente implica la "creación de una estrategia personal de información” y el desarrollo de destrezas de construcción de conocimiento, entre las que destacamos la capacidad para comprender un problema y seguir un conjunto de pasos valorando las herramientas que tenemos al alcance y las que debemos conseguir- y la capacidad para realizar juicios de valor informados (Gros, Contreras, 2006,109).

Pero, paralelamente a todo esto, potenciar el aprendizaje autónomo también se refiere a aceptar que, en ocasiones el trabajo es individual y que, precisamente por ello, es susceptible de mejora. Se relaciona directamente con el valor del esfuerzo, fundamental para poder afrontar los pequeños “contratiempos” o frustraciones” que inevitablemente se presentarán en el proceso de formación; se relaciona también con la capacidad de la persona para detectar cuando necesita ayuda y la muestra de confianza hacia los otros para llevar a cabo un trabajo cooperativo y/o colaborativo.

\section{Trabajo cooperativo y colaborativo}

Las tecnologías de la información y la comunicación, por sus características, constituyen un medio ideal para el desarrollo de ciertas competencias relacionadas con el aprendizaje autónomo y el trabajo cooperativo o, dicho de otro modo, con el estudio independiente y la realización de acciones apoyadas en el trabajo colaborativo. (Monereo, 2005; Cabero, 2006).

Si bien a primera vista pudiera parecer que son contrarios, el aprendizaje autónomo y el colaborativo son las dos caras de la misma moneda ya que favorecen que la persona

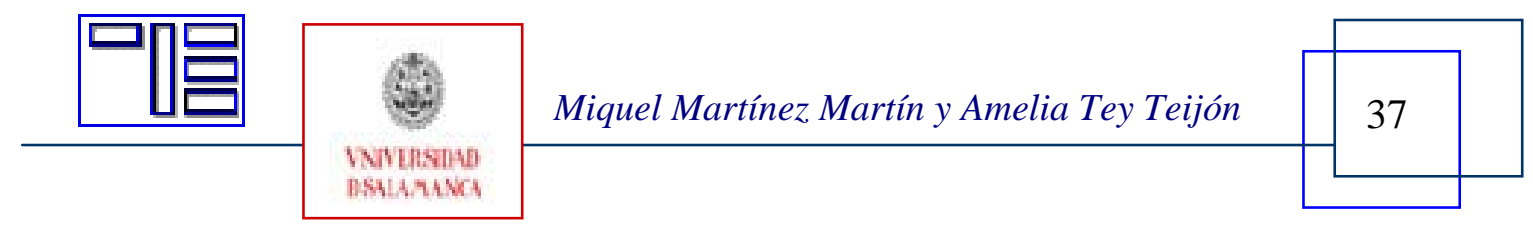


consiga el máximo desarrollo de sus potencialidades y construya conocimiento, en un entorno social. Permiten que la persona construya su autonomía en la interrelación y dependencia con los otros.

El aprendizaje autónomo no está reñido con el trabajo cooperativo y colaborativo, si bien hay quien afirma que incentivar el trabajo en equipo es una de las tareas más difíciles en el entorno virtual (Echevarría, 2000). No obstante, pueden proporcionar un punto de anclaje para referencias compartidas que se van construyendo a través de las actividades colaborativas, porque ayudan a "visibilizar" el entorno de colaboración favoreciendo el desarrollo de procesos metacognitivos (Garcia, 2007,)

Estamos convencidos que el desarrollo de las competencias éticas en la formación virtual debe fundamentarse en una concepción participativa del aprendizaje. (Gros, Contreras, 2006,109). La consideración de los otros en el desarrollo de aprendizaje autónomo supone la concienciación acerca de la existencia de otras personas en la red, en el mismo proceso de formación, con las que se puede contactar por ejemplo para debatir temas o pedir ayuda.

Una persona autónoma y colaboradora muestra niveles elevados de competencia ciudadana que le permite aprender a aprender, a trabajar en grupo, a comunicarse, a empatizar, a ser crítico y a proponerse metas tanto personales como comunitarias. (Monereo, 2005)

La implicación en la elaboración y/o resolución de proyectos colectivos "fuerza” a los participantes a utilizar las herramientas dialógicas para transmitir su punto de vista, conseguir la apertura hacia perspectivas diferentes -no sólo conociendo si no también comprendiendo la postura del otro-, dividir el trabajo -distributiva o equitativamente- y asumir su responsabilidad individual además de responsabilizarse colectivamente (Jonas, 1994). Se ha afirmado que la ciudadanía digital debe reunir los valores de honestidad, solidaridad y generosidad, tolerancia y respeto, responsabilidad y perseverancia (Eduteka, 2005). Son valores morales que consideramos fundamentales desde la perspectiva formativa de una universidad de calidad y comprometida con lo público y la formación de ciudadanía, y que Prats engloba bajo la responsabilidad como valor máximo, que relaciona con la sobriedad y la confianza. El autor identifica un ciudadano responsable como una persona sobria y confiada a la vez que confiable (Prats, 2005), rasgos básicos para el buen desarrollo del trabajo en equipo y cooperativo.

\section{5.- REFERENCIAS}

BAUMAN, Z (2000). Modernidad Líquida. Buenos Aires, Fondo de Cultura Económica.

BUXARRAIS, M.R.; TEY, A. (2005) Aprendizaje de competencias y formación de la ciudadanía en MARTÍNEZ, M.; PAYÀ, M. (Coords.) (2005) La formación de la ciuda-

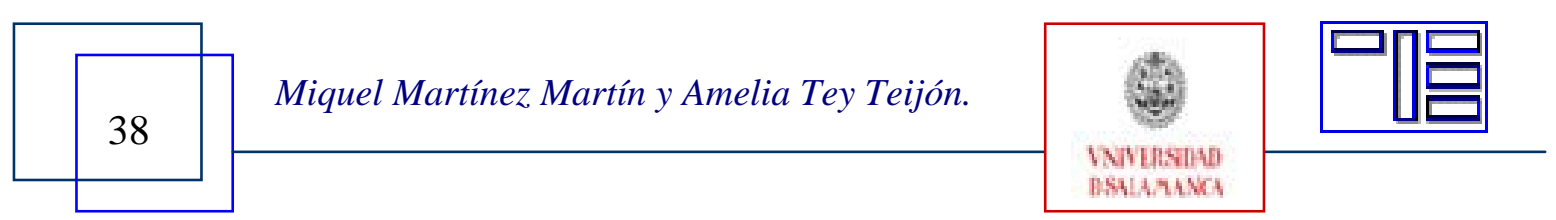


danía en el espacio europeo de educación superior. Madrid, Academia Europea de Ciencias y Artes. Pp 42.59.

CABERO, J (2006) Bases Pedagógicas del e-learning, en Revista de Universidad y Sociedad del Conocimiento, vol.3,1, abril.

COLL, C.; PALACIOS, J.; MARCHESI, A (compl.)(2001) Desarrollo psicológico y educación. Barcelona, Alianza editorial.

CROOK, CH. (1998) Ordenadores y aprendizaje colaborativo. Madrid: Morata.

DUART, J.M. (2003) Educar en valors als entorns virtuals d'aprenentatge: realitats i mites. http://www.uoc.edu/dt/20188/index.html. (última revisió 10 de juny de 2007)

ECHEVARRÍA, J. (2000) Educación y tecnologías telemáticas, en Revista Iberoamericana de Educación, 24, pp.17-35, Madrid, OEI.

EDUTEKA (2005) Ciudadanía digital. www.eduteka.org (última consulta: 18 de julio de 2007)

GARCIA,I. (2007) Aprender colaborando mediante las TIC en Aula. De Innovación Educativa, Monográfico El aprendizaje colaborativo a través de la red, 162, 51-58.

GROS, B.; CONTRERAS, D (2006). La alfabetización digital y el desarrollo de competencias ciudadanas, en Revista Iberoamericana de Educación, 42,103-127, Madrid, OEI.

HABERMAS,J. (1998) Conciencia moral y acción comunicativa. Barcelona, Ediciones Península.

JONAS. H. (1994). El principio de responsabilidad. Ensayo de una ética para la civilización tecnológica. Barcelona: Herder.

MARTINEZ, M. (2006). Formación para la ciudadanía y educación superior, en en Revista Iberoamericana de Educación, 42, 85-103, Madrid, OEI.

MARTINEZ, M.; HOYOS, G (Coords) (2006) La formación en valores en sociedades democráticas. Barcelona: Octaedro-OEI.

MARTÍNEZ, M.; PAYÀ, M. (Coords.) (2005) La formación de la ciudadanía en el espacio europeo de educación superior. Madrid, Academia Europea de Ciencias y Artes.

MARTÍNEZ, M.; BUXARRAIS, M.R.; ESTEBAN; BARA, F. (2002) "La universidad como espacio de aprendizaje ético" en Revista Iberoamericana de Educación, $\mathrm{n}^{\mathrm{a}} 29$, pp.17-42

MONERERO, C (coord.) (2005) Internet y competencias básicas. Barcelona, Graó.

NOGUERA, E (2004) Educació moral i en valors mitjançant projectes telemàtics. Tesis Doctoral.

PRATS, E.; BUXARRAIS,M.R.; TEY,A. (2004) Etica de la información. Barcelona, Ediuoc.

PRATS, E (2005). Redefinición de lo educativo en el cibercontexto: a propósito de las TIC como herramienta de inclusión, en Revista electrónica Teoría de la Educación, volumen 6 (1) (última consulta: 18 de julio de 2007)

Teleobservatorio Universitario de Docencia Virtual (Nettlab://http://netlab.urv.net)

SIGALÉS, C (2004) Formación universitaria y TIC: nuevos usos y nuevos roles, en Revista de Universidad y Sociedad del Conocimiento, vol 1. www.uoc.edu/rusc (última revisión: 25 de mayo 2007)

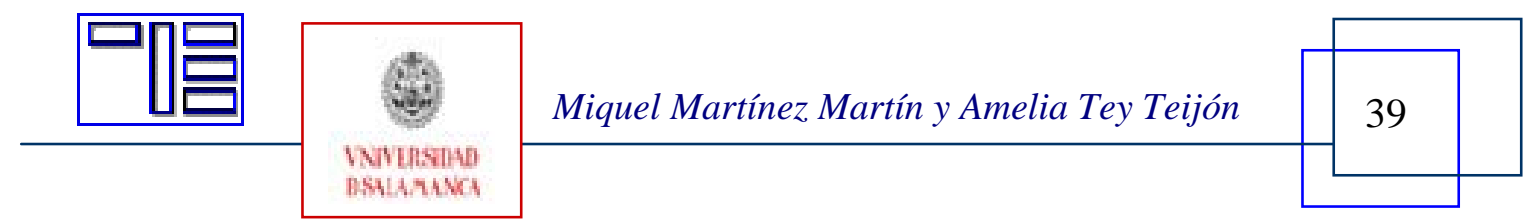


Revista Electrónica Teoría de la Educación.

Educación y Cultura en la Sociedad de la Información.

http://www.usal.es/teoriaeducacion

Vol. 9. No 1. Febrero 2008

TAYLOR, CH. (1994) Etica de la autenticidad. Barcelona, Paidós.

TEY, A (2004) Información y ética profesional, en PRATS, E.; BUXARRAIS, M.R.; TEY, A. Ética de la Información. Barcelona, UOC.

YUS, F. (2001) Ciberpragmática. Entre la compensación y el desconcierto” en http://www.cibersociedad.net (última revisión: 10 de julio de 2007)

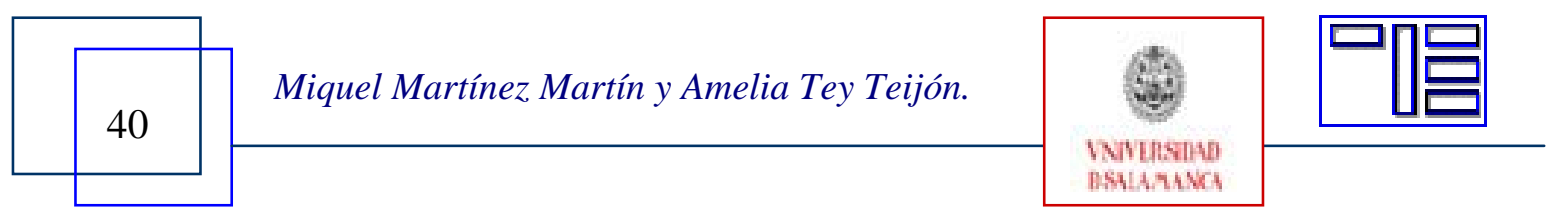

